International Mathematical Forum, 2, 2007, no. 30, 1469 - 1475

\title{
Random Structure Spaces
}

\author{
Junyu Gao \\ Department of Mathematics \\ Cangzhou Normal College \\ 061001, Hebei, P.R. China \\ Yongfu Su \\ Department of Mathematics \\ Tianjin Polytechnic University \\ 300160 Tianjin, P.R. China \\ suyongfu@gmail.com
}

\begin{abstract}
This paper present definition and basic concepts of random structure spaces, therefore, study relations of random metric spaces, random normed spaces, random inner product spaces and probabilistic metric spaces, probabilistic normed spaces, probabilistic inner product spaces. Finally, pose some questions for study or discussion.
\end{abstract}

Keywords: Random Structure Spaces; Probabilistic Metric Spaces; Probabilistic Normed Spaces; Probabilistic Inner Product Spaces

\section{Introduction And Preliminaries}

The probabilistic metric spaces was first introduced by K.Menger ${ }^{[1-3]}$ in 1942 , over past 60 years, mathematicians have done large amount of work in the theory of probabilistic metric spaces ${ }^{[4-6]}$.

Throughout this paper, $R$ denote real numbers set and $R^{+}$the nonnegative real numbers set. A mapping $F: R \rightarrow R^{+}$is called a distribution function if it is nondecreasing and left continuous with $\lim _{t \rightarrow-\infty} F(t)=0, \lim _{t \rightarrow+\infty} F(t)=$ 1. Throughout this paper, $D$ denote the all distribution functions set, $H(t)$ denote the specific distribution function which satisfying $H(0)=0$ and, for all $t>0, H(t)=1$.

Definition1.1 ${ }^{[1,4,5]}$ A Menger probabilistic metric space is a triple $(X, F, \Delta)$, where $X$ is an abstract set and $F$ is a mapping of $X \times X \longrightarrow D$, i.e, $F$ associates a distribution function $F(x, y)$ with every pair $(x, y)$ of points in $X$. We shall denote the distribution function $F(x, y)$ by $F_{x, y}$, whence the symbol 
$F_{x, y}(t)$ will denote the value of $F_{x, y}$ for the real argument $t$. The distribution functions are assumed to satisfy the following conditions:

$(P M-1) F_{x, y}(0)=0, \forall x, y \in X$

$(P M-2) F_{x, y}(t)=F_{y, x}(t), \forall x, y \in X, t \in R$;

$(P M-3) F_{x, y}(t)=H(t) \Longleftrightarrow x=y$;

$(P M-4) F_{x, y}\left(t_{1}+t_{2}\right) \geq \triangle\left(F_{x, z}\left(t_{1}\right), F_{z, y}\left(t_{2}\right)\right), \forall x, y, z \in X, t_{1}, t_{2} \geq 0$,

where $\triangle:[0,1] \times[0,1] \longrightarrow[0,1]$ is said to be triangle norm which satisfying the following conditions:

$$
\begin{aligned}
& (\Delta-1) \quad 0 \leq \Delta(a, b) \leq 1 ; \\
& (\Delta-2) c \geq a, d \geq b \Rightarrow \Delta(c, d) \geq \Delta(a, b) ; \\
& (\Delta-3) \Delta(a, b)=\Delta(b, a) ; \\
& (\Delta-4) \Delta(1,1)=1 ; \\
& (\Delta-5) \Delta(a, 1)>0, \forall a>0 .
\end{aligned}
$$

Definition 1.2 ${ }^{[4,5]}$ A Menger probabilistic normed space is a triple $(X, F, \Delta)$, where $X$ is a real linear space, and $F$ is a mapping of $X \longrightarrow D$, i.e, $F$ associates a distribution function $F(x)$ with every pair $x$ of points in $X$. We shall denote the distribution function $F(x)$ by $F_{x}$, whence the symbol $F_{x}(t)$ will denote the value of $F_{x}$ for the real argument $t$. The distribution functions are assumed to satisfy the following conditions:

$$
\begin{aligned}
& (P N-1) F_{x}(0)=0, \forall x \in X ; \\
& (P N-2) F_{x}(t)=H(t) \Longleftrightarrow x=0 ; \\
& (P N-3) F_{\alpha x}(t)=F_{x}\left(\frac{t}{\alpha}\right), \forall x \in X, \alpha \neq 0 ; \\
& (P N-4) F_{x+y}\left(t_{1}+t_{2}\right) \geq \triangle\left(F_{x}\left(t_{1}\right), F_{y}\left(t_{2}\right)\right), \forall t_{1}, t_{2} \geq 0, x, y \in X .
\end{aligned}
$$

Definition 1.3 ${ }^{[4,6]}$ A triple $(X, F, *)$ is called a probabilistic inner product space if, $X$ is a real linear apace, $F$ is a mapping from $X \times X$ into $D$ satisfying the following conditions, where we denote $F(x, y)$ by $F_{x, y}(t)$ will present the value of $F_{x, y}$ at $t \in R$ :

$(P I-1) F_{x, x}(0)=0, \forall x \in E$;

$(P I-2) F_{x, y}=F_{y, x}, \forall x, y \in E$;

$(P I-3) F_{x, x}(t)=H(t)$ if and only if $x=0$;

$(P I-4)$ for all $x, y \in X$ and $\alpha \in R$, when $\alpha>0$ then $F_{\alpha x, y}(t)=F_{x, y}\left(\frac{t}{\alpha}\right)$, when $\alpha=0$ then $F_{\alpha x, y}(t)=H(t)$, when $\alpha<0$ then $F_{\alpha x, y}(t)=1-F_{x, y}\left(\frac{t}{\alpha}+\right)$; $(P I-5)$ If $x, y$ are linearly independent, then

$$
F_{x+y, z}(t)=\left(F_{x, z} * F_{z, y}\right)(t)
$$

Where

$$
\left(F_{x, z} * F_{z, y}\right)(t)=\int_{-\infty}^{+\infty} F_{x, z}(t-u) d F_{z, y}(u)
$$

In this paper, now, we give the following definition of random structure space. 
Definition 1.4. Let $E$ be a nonempty set, $(S, \bar{\Re})$ be a measurable space, where $S$ consist of elements which have connections with set $E,(\Omega, \Re, P)$ be a completely probabilistic measure space and $f: \Omega \rightarrow S$ be a measurable mapping with respect to $\Re$ and $\bar{\Re}$. Then the quadruplet $(E, S, \Omega, f)$ is called a random structure space on $E$.

Therefore, we give the following definitions of random topological space, random metric space, random normed space and random inner product space.

Definition 1.5. Let $(E, S, \Omega, f)$ be a random structure space.

(i) If $S$ is a family of topologies on $E$, then $(E, S, \Omega, f)$ is called a random topological space;

(ii) If $S$ is a family of metric on $E$, then $(E, S, \Omega, f)$ is called a random metric space (shortly, RM- space);

(iii) If $E$ is a vector space and $S$ is a family of norms on $E$, then $(E, S, \Omega, f)$ is called a random normed space (shortly, RN-space);

(iv) If $E$ is a vector space and $S$ is a family of inner products on $E$, then $(E, S, \Omega, f)$ is called a random inner product space (shortly, RI-space).

\section{Main Results}

Definition 2.1. Let $(E, S, \Omega, f)$ be a $R M$-space. If, for every $x, y \in E$ and all real number $t \in R,\{d \in S: d(x, y)<t\}$ is measurable subset in $S$, then $(E, S, \Omega, f)$ is called a normal $R M$-space.

Theorem 2.1. Let $(E, S, \Omega, f)$ be a normal $R M$-space. For every $x, y \in E$ and real number $t \in R$, define

$$
F_{x, y}(t)=P\left\{f^{-1}\{d \in S: d(x, y)<t\}\right\} .
$$

Then $\left(E, F_{x, y}(t), \Delta\right)$ is an Menger probabilistic metric space, where $\Delta(a, b)$ $=\max \{a+b-1,0\}$.

Proof. It is easily to see that $F_{x, y}(t)$ is a distribution function which satisfies the following conditions:

$(\mathrm{PM}-1) F_{x, y}(0)=0, \forall x, y \in E$;

$(\mathrm{PM}-2) F_{x, y}(t)=F_{y, x}(t), \forall x, y \in E$;

(PM-3) $F_{x, y}(t)=H(t)$ if and only if $x=y$,

where $H(t)$ is a special distribution function, when $t \leq 0$ then $H(t)=0$, when $t>0$ then $H(t)=1$.

Next we shall prove that the Menger triangle inequality (PM-4) holds. Indeed, for every $x, y, z \in E$ and real numbers $t_{1}, t_{2} \geq 0$, we have

$$
\begin{gathered}
F_{x, y}\left(t_{1}+t_{2}\right)=P\left\{f^{-1}\left(\left\{d \in S: d(x, y)<\left(t_{1}+t_{2}\right)\right\}\right)\right\} \\
\geq P\left\{f^{-1}\left(\left\{d \in S: d(x, z)<t_{1}, d(z, y)<t_{2}\right\}\right)\right\} \\
=P\left\{f^{-1}\left(\left\{d \in S: d(x, z)<t_{1}\right\} \cap\left\{d \in S: d(z, y)<t_{2}\right\}\right)\right\} \\
\left.=P\left\{f^{-1}\left(\left\{d \in S: d(x, z)<t_{1}\right\}\right\}\right)\right\}+P\left\{f^{-1}\left(\left\{d \in S: d(y, z)<t_{2}\right\}\right)\right\} \\
-P\left\{f^{-1}\left(\left\{d \in S: d(x, z)<t_{1}\right\} \cup\left\{d \in S: d(y, z)<t_{2}\right\}\right)\right\}
\end{gathered}
$$




$$
\geq F_{x, z}\left(t_{1}\right)+F_{z, y}\left(t_{2}\right)-1
$$

which implies that

$$
F_{x, y}\left(t_{1}+t_{2}\right) \geq \Delta\left(F_{x, z}\left(t_{1}\right), F_{z, y}\left(t_{2}\right)\right),
$$

and hence,$\left(E, F_{x, y}(t), \Delta\right)$ is a Menger probabilistic metric space. This completes the proof of Theorem 2.1.1.

Definition 2.2. Let $(E, S, \Omega, f)$ be a normal $R M$-space, and for $x, y \in E$ and all real number $t \in R$, let

$$
F_{x, y}(t)=P\left\{f^{-1}(\{d \in S: d(x, y)<t\})\right\} .
$$

If $\left(E, F_{x, y}(t), \Delta\right)$ is a Menger probabilistic metric space, then it is called an orbit space of $(E, S, \Omega, f)$.

Theorem 2.2. Let $\left(E, F_{x, y}\right.$, min) be a Menger probabilistic metric space which satisfying the condition

$$
\sup \left\{t \in R, F_{x, y}(t)=0\right\}=0 \text {, if } x \neq y .
$$

Then there exists a normal $R M$-space $(E, S, \Omega, f)$ such that $\left(E, F_{x, y}(t)\right.$, min) is an orbit space of $(E, S, \Omega, f)$.

Proof. For every $p \in[0,1)$ and $x, y \in E$, we define a mapping $d_{p}: E \times E \rightarrow$ $R$ as follows:

$$
d_{p}(x, y)=\sup \left\{t \in R: F_{x, y}(t) \leq p\right\},
$$

we shall prove that $d_{p}$ is a metric on $E$. In fact, $d_{p}(x, y) \geq 0$ and $d_{p}(x, y)=$ $d_{p}(y, x)$ are obvious. It follows from condition of $\left(E, F_{x, y}, \min \right)$ that, $d_{p}(x, y)$ $=0$ if and only if $x=y$. If the triangle inequality does not holds, then there exists $x, y, z \in E$ and $\epsilon>0$ such that

$$
d_{p}(x, y)=d_{p}(x, z)+d_{p}(z, y)+2 \epsilon .
$$

It follows that

$$
\begin{gathered}
p=F_{x, y}\left(d_{p}(x, y)\right)=F_{x, y}\left(d_{p}(x, z)+d_{p}(z, y)+2 \epsilon\right) \\
\geq \min \left\{F_{x, z}\left(d_{p}(x, z)+\epsilon\right), F_{z, y}\left(d_{p}(z, y)+\epsilon\right)\right\}>\min \{p, p\}=p,
\end{gathered}
$$

this is a contradiction.

Let $S=\left\{d_{p}: p \in[0,1)\right\}$ and $\Omega=[0,1)$ be a Lebesgue probabilistic measure space. Let $f: \Omega \rightarrow S$ be a mapping such that $f(p)=d_{p}$ for every $p \in \Omega$. Since $f$ is a one to one, then $f$ induces a measure on $S$, thus $(E, S, \Omega, f)$ is a $R M$-space. For every $x, y \in E$ and $t \in R$, we have

$$
\left\{d_{p} \in S: d_{p}(x, y)<t\right\}=\left\{d_{p} \in S: F_{x, y}(t)>p\right\}
$$

which implies that $\left\{d_{p} \in S: d_{p}(x, y)<t\right\}$ is a measureable set and so $(E, S, \Omega, f)$ is a normal $R M$-space. Finally, we prove that $\left(E, F_{x, y}(t)\right.$, min) is an orbit space of $(E, S, \Omega, f)$, indeed,

$$
F_{x, y}(t)=P\left\{f^{-1}\left(\left\{d_{p} \in S: F_{x, y}(t)>p\right\}\right)\right\}
$$




$$
=P\left\{f^{-1}\left(\left\{d_{p} \in S: d_{p}(x, y)<(t)\right\}\right)\right\},
$$

where $P$ is a Lebesgue measure on $\Omega=[0,1)$. This completes the proof.

Theorem 2.3. Let $(E, S, \Omega, f)$ be a normal $R N$-space. For every $x \in E$ and real number $t \in R$, define

$$
F_{x}(t)=P\left\{f^{-1}\{d \in S: d(x)<t\}\right\} .
$$

Then $\left(E, F_{x}(t), \Delta\right)$ is an Menger probabilistic normed space, where $\Delta(a, b)$ $=\max \{a+b-1,0\}$.

The proof of theorem2.3 is similar to theorem 2.1.

Theorem 2.4. Let $\left(E, F_{x}, \min \right)$ be a Menger probabilistic normed space which satisfying the condition

$$
\sup \left\{t \in R, F_{x}(t)=0\right\}=0, \text { if } x \neq 0 .
$$

Then there exists a normal $R N$-space $(E, S, \Omega, f)$ such that $\left(E, F_{x}(t)\right.$, min $)$ is an orbit space of $(E, S, \Omega, f)$.

The proof of theorem2.4 is similar to theorem 2.2.

Definition 2.3. Let $(E, S, \Omega, f)$ be a $R I$-space. If, for every $x, y \in E$ and all real numbers $t \in R,\{I \in S: I(x, y)<t\}$ is measurable subset in $S$, then $(E, S, \Omega, f)$ is called a normal $R I$-space.

Lemma Let $(E, S, \Omega, f)$ be a $R I$-space. For every $x, y, z \in E$ and real number $t \in R$, we denote $A, B_{n}, B^{n}$ by

$$
\begin{aligned}
A & =\{I \in S: I(x+y, z)<t\}=\{I \in S: I(x, z)+I(z, y)<t\}, \\
B_{n} & =\bigcup_{m \in Z}\left\{I \in S: \frac{m}{n} \leq I(z, y)<\frac{m+1}{n}, I(x, z)<t-\frac{m+1}{n}\right\}, n=1,2,3, \ldots \\
B^{n} & =\bigcup_{m \in Z}\left\{I \in S: \frac{m}{n} \leq I(z, y)<\frac{m+1}{n}, I(x, z)<t-\frac{m}{n}\right\}, n=1,2,3, \ldots
\end{aligned}
$$

Then $B_{n} \subseteq A \subseteq B^{n}$, where $Z$ denote the integral numbers set.

Proof If $I \in B_{n}$, then there exists $m \in Z$ such that

$$
\frac{m}{n} \leq I(z, y)<\frac{m+1}{n}, I(x, z)<t-\frac{m+1}{n}
$$

which implies that $I(x, z)+I(z, y)<t$, so that $B_{n} \subseteq A$.

Other hand, if $I \in A$ then $I(x, z)+I(z, y)<t$, we can taking $m \in Z$ so that

which leads to

$$
\frac{m}{n} \leq I(z, y) \leq \frac{m+1}{n}
$$

$$
I(x, z)<t-I(z, y)<t-\frac{m}{n}
$$

then $A \subseteq B^{n}$, this complete the proof of lemma. 
Theorem 2.5. Let $(E, S, \Omega, f)$ be a normal $R I$-space. For every $x, y \in E$ and real number $t \in R$, define

$$
F_{x, y}(t)=P\left\{f^{-1}\{I \in S: I(x, y)<t\}\right\} .
$$

Then $\left(E, F_{x, y}(t)\right.$ is a probabilistic inner product space.

Proof The conditions $(P I-1),(P I-2)(P I-3)$ of probabilistic inner product space are easily to prove, we will prove the condition $(P I-4)$ in following

When $\alpha>0$, then

$$
\begin{aligned}
& F_{\alpha x, y}(t)=P\left\{f^{-1}\{I \in S: I(\alpha x, y)<t\}\right\} \\
= & P\left\{f^{-1}\left\{I \in S: I(x, y)<\frac{t}{\alpha}\right\}\right\}=F_{x, y}\left(\frac{t}{\alpha}\right) .
\end{aligned}
$$

When $\alpha=0$, then

$$
F_{\alpha x, y}(t)=P\left\{f^{-1}\{I \in S: I(0, y)<t\}\right\}=H(t) .
$$

When $\alpha<0$, then

$$
\begin{gathered}
F_{\alpha x, y}(t)=P\left\{f^{-1}\{I \in S: I(\alpha x, y)<t\}\right\} \\
=P\left\{f^{-1}\left\{I \in S: I(x, y)>\frac{t}{\alpha}\right\}\right\} \\
=1-P\left\{f^{-1}\left\{I \in S: I(x, y) \leq \frac{t}{\alpha}\right\}\right\}=1-F_{x, y}\left(\frac{t}{\alpha}+\right) .
\end{gathered}
$$

This complete the proof of condition $(P I-4)$. Finally, we will prove the condition $(P I-5)$ of Random inner product spaces.

Since the $(E, S, \Omega, f)$ is normal $R I$-space, so that $A, B_{n}, B^{n}$ are measurable subset of $S$. It follows from lemma that

$$
P\left\{f^{-1}\left(B_{n}\right)\right\} \leq P\left\{f^{-1}(A)\right\} \leq P\left\{f^{-1}\left(B^{n}\right)\right\},
$$

where $P$ is probabilistic measure on the $\Omega$. On the other hand, we have

$$
\begin{gathered}
P\left\{f^{-1}\left(B_{n}\right)\right\}=\sum_{m \in Z} P\left\{f^{-1}\left\{I \in S: \frac{m}{n} \leq I(z, y)<\frac{m+1}{n}, I(x, z)<t-\frac{m+1}{n}\right\}\right\} \\
=\sum_{m \in Z} P\left\{f^{-1}\left\{I \in S: \frac{m}{n} \leq I(z, y)<\frac{m+1}{n}\right\}\right\} \cdot P\left\{f^{-1}\left\{I(x, z)<t-\frac{m+1}{n}\right\}\right\} \\
=\sum_{m \in Z}\left[F_{z, y}\left(\frac{m+1}{n}\right)-F_{z, y}\left(\frac{m}{n}\right)\right] F_{x, z}\left(t-\frac{m+1}{n}\right) .
\end{gathered}
$$

Thus

$$
\begin{aligned}
\lim _{n \rightarrow \infty} P\left\{f^{-1}\left(B_{n}\right)\right\} & =\lim _{n \rightarrow \infty} \sum_{m \in Z}\left[F_{z, y}\left(\frac{m+1}{n}\right)-F_{z, y}\left(\frac{m}{n}\right)\right] F_{x, z}\left(t-\frac{m+1}{n}\right) . \\
& =\int_{-\infty}^{+\infty} F_{x, z}(t-u) d F_{z, y}(u),
\end{aligned}
$$


By a same way, we can obtain

$$
\lim _{n \rightarrow \infty} P\left\{f^{-1}\left(B^{n}\right)\right\}=\int_{-\infty}^{+\infty} F_{x, z}(t-u) d F_{z, y}(u) .
$$

Combining (1), (2) and (3) we obtain

$$
\lim _{n \rightarrow \infty} P\left\{f^{-1}(A)\right\}=\int_{-\infty}^{+\infty} F_{x, z}(t-u) d F_{z, y}(u) .
$$

Thus we obtain

$$
F_{x+y, z}(t)=\int_{-\infty}^{+\infty} F_{x, z}(t-u) d F_{z, y}(u) .
$$

This complete the proof of theorem2.5.

Remark The random variables and random elements are important and basic concepts of probabilistic theory and random functional analysis. However, random structures or random systems are even more important which have a widespread and profound influence for the applications. In the natural world, there exists many backgrounds and problems of application of random structure spaces. Therefore, the research and establish of theory of random structure spaces is very important and necessary.

Finally, we pose the following questions for study or discussion:

(1) The probabilistic distribution of random structure;

(2) The expectation and variance of random structure;

(3) The stability of random structure;

(4) The limit theory of random structure;

(5) The process of random structure;

(6) The study of random topological spaces;

(7) The theory of random structure spaces and science of systems.

\section{REFERENCES}

1. K.Menger, Statistical metric,Proc. Nat. Acad. of Sci. U.S.A 28(1942),535-537.

2. K.Menger, Probabilistic theories of relations, Proc. Nat. Acad. of Sci. U.S.A 37(1951),178-180.

3. K.Menger, Probabilistic geometry, Proc. Nat. Acad. of Sci. U.S.A 37(1951),226-229.

4. Shin-Sen Chang, Yeol Je Cho and Shin Min Kang, Probabilitic metric spaces and nonlinear operator theory, Sichuan University Press (1994).

5. Schweizer. B, Sklar.A, Probabilitic metric spaces, Elsevier-North Holland,New York (1983).

6. Yongfu $\mathrm{Su}$, On the probabilitic structure spaces, Stochastic Analysis and Applicatons, $2(2002), 149-156$.

\section{Received: September 2, 2006}

